Nervenarzt 2016 $87: 1012-1016$

DOI 10.1007/s00115-016-0174-1

Online publiziert: 16. August 2016

(c) Springer-Verlag Berlin Heidelberg 2016

CrossMark

\title{
H. Helmchen
}

Klinik für Psychiatrie und Psychotherapie, Charité - Universitätsmedizin Berlin, Campus Benjamin Franklin, Berlin, Deutschland

\section{Psychiater zwischen Schweige- und Offenbarungspflicht} Nervenheilkunde (DGPPN) mit einer Stellungnahme, in der sie Spekulationen über psychiatrische Diagnosen und die Gefährlichkeit psychischer Krankheiten als diskriminierend und stigmatisierend zurückwies und betonte, dass eine geforderte „Meldepflicht für psychische Erkrankungen (wie in der Zeit des Nationalsozialismus) und das Durchbrechen der ärztlichen Schweigepflicht ... die Angst und die Scham der von psychischer Erkrankung Betroffenen erhöhen und eine frühzeitige sachgerechte $\mathrm{Be}$ handlung verhindern wird" [3].

Mit ähnlicher Zielsetzung hat der am 13.3.2016 publizierte Bericht der französischen BEA-Untersuchungskommission nach sorgfältiger und angemessener Abwägung zwischen Schweigepflicht und Offenbarungspflicht keine wesentliche Änderung des Umgangs mit der ärztlichen Schweigepflicht empfohlen, wohl aber eine internationale Vereinheitlichung (und Präzisierung) der Kriterien zur ärztlichen Offenbarungspflicht vorgeschlagen [2].

Die folgenden Bemerkungen sollen anregen, sich auf die Umsetzung dieser Empfehlungen vorzubereiten, sind aber vielleicht auch von allgemeinerem Interesse, da Psychiater nicht nur bei Piloten vor solche Abwägungen geraten können, sondern auch bei Menschen aus anderen Arbeitsfeldern mit personenbezogenem Gefährdungspotenzial - von Mitarbeitern aus der Atom- und Chemieindustrie über Lokomotivführer bis zu Bus- und Taxifahrern. Immerhin wurde jetzt eine Klage gegen eine behandelnde Ärztin wegen Unterlassung einer solchen Meldung eingereicht.

\section{Schweigepflicht}

Denn die Schweigepflicht ist die Voraussetzung dafür, dass der Patient sich gegenüber dem Arzt öffnet und ihm auch Intimstes im Vertrauen darauf berichtet, dass der Arzt alles für sich behält und gegenüber Dritten schweigt. Nur so kann der Psychiater die Informationen erhalten, die ihm eine zutreffende Beurteilung des psychischen Krankheitszustands und möglicher Gefährdungen ermöglichen.

\section{Offenbarungsbefugnis und Offenbarungspflicht}

Gleichwohl gibt es Situationen, die den Arzt zu der Erwägung zwingen, ob er die Schweigepflicht brechen muss. Denn der Arzt hat eine Offenbarungsbefugnis, wenn er nach gewissenhafter Abwägung der involvierten Rechtsgüter eine erhebliche Gefährdung eines gegenüber der Verschwiegenheit höheren Rechtsguts sieht, oder sogar eine Offenbarungspflicht bei unmittelbar drohender Lebensgefährdung von Menschen. Dabei geht es um die Frage, wie ein von einem psychisch Kranken ausgehendes lebensgefährdendes Risiko am besten minimiert werden kann. Folgen unterschiedlicher Lösungen dieser Pflichtenkollision zwischen Schweigen und Offenbaren sollen mit einigen Falldarstellungen illustriert werden.

Die Schweigepflicht des Arztes ist nicht nur seit Hippokrates ethisch begründet, sondern auch durch das geltende Recht hochrangig geschützt, so durch Artikel 2 Abs. 1 des Grundgesetzes, weiter durch $\$ 9$ der Berufsordnung für Ärzte und schließlich als vertragliche Nebenpflicht aus dem Behandlungsvertrag sowie auch durch $₫ 203$ des Strafgesetzbuchs (StGB) und indirekt durch Datenschutzgesetze.

\section{Das Tarasoff-Urteil und seine Folgen}

Die Offenbarungspflicht wurde für zahlreiche Bundesstaaten der USA im sog. Tarasoff-Urteil des Supreme Court von Kalifornien 1976 festgeschrieben. Ein Psychotherapeut hatte die Absicht seines Patienten für sich behalten, eine Frau 
namens Tatjana Tarasoff zu töten, da sie die Zuneigung des Patienten zurückgewiesen hatte; der Psychotherapeut wurde nach der ausgeführten Tat von den Hinterbliebenen mit dem Argument erfolgreich verklagt, dass er sie hätte warnen müssen [10].

Seitdem werden dieses richtungsweisende Urteil und seine widersprüchlichen Folgen für die amerikanische Legislative und Judikative sowie die psychiatrische Praxis kontrovers diskutiert:

Eine Folge ist die dadurch entstandene Vielfalt unterschiedlicher rechtlicher Regelungen, da eine Reihe von US-amerikanischen Staaten eine solche Offenbarungspflicht gesetzlich fixiert hat, während diese in weiteren Staaten nur auf - in einzelnen Aspekten unterschiedliche - Gerichtsurteile gestützt wird. Wieder andere Staaten überlassen die Entscheidung dem gewissenhaften Ermessen des Arztes oder sehen gar keine Regelungen vor. Auch unterscheiden sich Konsequenzen der Offenbarungspflicht, nämlich entweder das potenzielle Opfer warnen („duty to warn“) oder aber durch andere Maßnahmen wie die Zwangseinweisung des Gefährders schützen zu müssen („duty to protect“). Ein spezielles Problem ergibt sich, wenn das potenziell gefährdende Verhalten eines Menschen mit Persönlichkeitsstörung besonders schwer zu prognostizieren ist [6].

Eine weitere Folge sind autoprotektive Reaktionen der Therapeuten zum Schutz vor strafrechtlicher Verfolgung oder Schadensersatzforderungen, indem beispielsweise Psychiater und Psychologen Patienten radikal über die jeweiligen Offenbarungsvorschriften aufklären mit dem Effekt, dass Patienten nicht mehr offen mit dem Therapeuten kommunizieren [8, 9, 11]. Eine empirische Untersuchung solcher Konsequenzen des Tarasoff-Urteils fand eine Zunahme von Homiziden in den USA um 8,9\% und kam zu dem Schluss, dass die gesetzliche Pflicht zur Offenbarung fallen gelassen werden solle [5].

\section{Erfordernisse, Kriterien und Risiken einer Offenbarung}

Im Laufe dieser Kontroverse wurden die Bedingungen einer Offenbarung präzi- siert und von einer „duty to warn“ $\mathrm{zu}$ einer „duty to protect“verändert: Erstens soll eine ausreichende Erhebung (und Dokumentation) der für eine Gefährdungsbeurteilung notwendigen Befunde erfolgen.Zweitens sind Maßnahmen zum Schutz konkreter potenzieller Opfer zu treffen. So erscheint etwa eine Klinikeinweisung des psychisch kranken Gefährders oder seine Verlegung auf eine geschlossene Station akzeptabler als ein Bruch der Schweigepflicht durch Warnung potenzieller Opfer. Drittens sollen die getroffenen Sicherungsmaßnahmen auf ihre Wirksamkeit und Notwendigkeit kontinuierlich überprüft werden [1].

\section{》) Eine Klinikeinweisung erscheint akzeptabler als die Warnung potenzieller Opfer}

Als Kriterien eines offenbarungspflichtigen Risikos sind im US-amerikanischen Recht vor allem die konkrete Identifizierbarkeit potenzieller Opfer und die Unmittelbarkeit der drohenden Lebensgefährdung genannt sowie auch die Größe des Risikos, wobei unklar bleibt, ob mit Größe die Intensität oder der Umfang der Gefährdung gemeint ist.

Dass Psychiater ein gerade bei psychisch Kranken oft labiles Vertrauensverhältnis nur im äußersten Notfall belasten wollen, ist verständlich, besonders wenn sie hoffen, den Patienten durch eine vertrauensvolle Führung davon abhalten zu können, eine geäußerte Absicht auch zu realisieren. Dabei müssen sie jedoch sensibel für das Risiko bleiben, die Absicht des Patienten nicht klar genug zu erfahren und deren Realisierungswahrscheinlichkeit unzutreffend zu beurteilen. Die Einschätzung der Eindeutigkeit und insbesondere der Erheblichkeit der Absichten des Patienten muss umso dezidierter wahrgenommen werden, je schwerwiegender das potenzielle Risiko einer Fehlhandlung des psychisch Kranken ist. Ein Beispiel:

Im Jahr 1972 musste der US-amerikanische Präsidentschaftskandidat George McGovern seinen Kandidaten für das Vizepräsidentenamt Thomas Eagleton entlassen, nachdem er durch einen anonymen Anruf erfahren hatte, dass Eagleton
Jahre zuvor in psychiatrischer Behandlung gewesen war. Dass ein ehemals depressiver Mensch als Vizepräsident in der Hochzeit des Kalten Krieges Zugang zum „Knopf“ („finger on the button“) haben könnte, mit dem sich ein Atomkrieg auslösen lässt, wurde als zu großes Sicherheitsrisiko angesehen - nach Rücksprache von McGovern mit zwei Psychiatern, die Eagleton von ihrer Schweigepflicht entbunden hatte [7].

Der Fall zeigt den Einfluss, den eine psychiatrische Vorgeschichte auf die berufliche Situation eines Menschen haben kann - nicht wegen der Depression selbst, denn Eagleton arbeitete danach fast 20 Jahre qualifiziert als Senator und Professor weiter, sondern wegen der womöglich durch stigmatisierende Vorurteile oder politische Absichten beeinflussten Information darüber. Weiterhin illustriert der Fall die Abhängigkeit der Abwägungen zwischen Verschwiegenheit und Offenbarungspflicht von der Höhe des Risikos. Übrigens wird seitdem in den USA der medizinische und biografische Hintergrund von Kandidaten für wichtige Ämter obligatorisch recherchiert.

\section{Nutzen-Risiko-Relation von Schweigepflicht vs. Offenbarung}

Sind die Einschätzungen von Eindeutigkeit und Erheblichkeit des Risikos oft schon schwierig genug, so erst recht die der Relation des Nutzens der Schweigepflicht, also der Erhaltung des Vertrauensverhältnisses und damit der Möglichkeit, den Patienten tatverhindernd zu beeinflussen, zum Risiko des Bruchs der Schweigepflicht und damit des Behandlungsabbruchs, der den meist ambivalenten Patienten sich selbst überlassen würde oder ihn bei einem anderen Arzt eher seine Symptomatik verschweigen lässt wie die zitierten amerikanischen Erfahrungen zeigen.

\section{Zurück zur Germanwings- Katastrophe}

Im 117 Seiten umfassenden, ebenso detaillierten wie sorgfältigen Bericht der französischen Behörde für Sicherheits- 
untersuchungen in der zivilen Luftfahrt (BEA) zu Unfallursachen wie vor allem zu möglichen Sicherheitsverbesserungen werden neben vielen Fakten zum konkreten Fall auch allgemeine Fakten festgestellt:

Die Rekrutierung potenzieller Piloten folgt einer strengen Auswahl: Lufthansa hat beispielsweise 2008 von 6530 Bewerbern nur 384, also knapp $6 \%$ zur Pilotenausbildung zugelassen.

Alle Piloten müssen jährlich ihre Flugtauglichkeitszeugnisse nach entsprechenden medizinischen Untersuchungen einschließlich psychiatrischer Beurteilungen erneuern lassen. In Norwegen etwa werden pro Jahr ca. 3000 Flugtauglichkeitszeugnisse ausgestellt, verlängert oder erneuert und rund 60 , d.h. ca. $2 \%$ abgelehnt ([2], S. 74).

Trotzdem ist der Germanwings-Unfall kein Einzelfall: Seit 1980 wurden weitere 12 Unfälle bekannt, bei denen das Flugzeug vom Piloten oder Kopiloten bewusst ab vom Kurs oder zum Absturz gebracht worden war - aus unbekannten Gründen oder infolge akut aufgetretener psychischer Störungen des Piloten (Angstzustände, Drogeneinnahme, wahnhafte Verwirrtheit; [2], S. 80 f.). Aber dieses Risiko ist bei aller bestürzenden Tragik des Einzelfalls im Verhältnis zu weltweit derzeit knapp 40 Mio. Flügen pro Jahr verschwindend klein, jedenfalls sehr viel kleiner als das von vermutlich suizidal verursachten Autounfällen. Ein völliger Ausschluss des Risikos scheint unmöglich zu sein, selbst nicht durch die "Zwei-Personen-Regel“ (s. unten), wie drei der 12 BEA-Fälle deutlich machen ([2], S. 81 f.).

Eine Umfrage der BEA zeigt, dass das „Fehlen von klaren Definitionen für die Begriffe , bevorstehende Gefahr ${ }^{c}$ und ,Bedrohung der öffentlichen Sicherheit' die Ärzte dazu bringt, einen eher konservativen Ansatz zu verfolgen. Sie werden medizinische Informationen nicht an Behörden weiter geben, es sei denn es gibt eine offensichtliche, eindeutige und unmittelbare Bedrohung eines Dritten oder des Patienten selbst. Sie nehmen eine solche Haltung nicht nur ein, weil sie das Vertrauen ihrer Patienten erhalten wollen, sondern auch, weil sie fürchten angeklagt zu werden, Sanktionen durch die
Justiz ausgesetzt zu werden und/oder das Recht verlieren könnten, zu praktizieren “ ([2], S. 102).

\section{Maßnahmen zur Erhöhung der Sicherheit}

In Deutschland ist die Güterabwägung zwischen Schweige- und Offenbarungspflicht dem pflichtgemäßen und gewissenhaften Ermessen des Arztes überlassen. Zahlen darüber, wie häufig überhaupt Ärzte die Schweigepflicht wegen einer als schwerwiegender angesehenen Offenbarungspflicht durchbrochen haben, sind nicht bekannt. (Ich erinnere mich an einen einzigen Fall in meiner 60-jährigen klinischen Tätigkeit.)

Will man Gefährdungen durch lebensgefährdende Akte psychisch Kranker minimieren, erscheinen Maßnahmen, die es dem psychisch Kranken ermöglichen, sich zu öffnen und Vertrauen $\mathrm{zu}$ entwickeln, wirksamer als die Offenbarung der Gefährdung, etwa wenn „in Deutschland, wie in den meisten europäischen Staaten, ... eine Depression ein klarer Grund dafür [ist] einen Piloten für fluguntauglich zu erklären. Es gibt Hinweise, dass Berufspiloten eine Medikation ablehnen, weil sie dann nicht mehr fliegen dürften. Es gibt auch Hinweise, dass Piloten Antidepressiva einnehmen, ohne dies den Luftfahrtbehörden zu melden, weil sie weiterhin fliegen wollen." So war nur bei 3 von 61 Piloten, bei denen „SSRI Medikamentenrückstände gefunden wurden, nachdem sie in den USA zwischen 1990 und 2001 bei Flugzeugunfällen tödlich verunglückt sind", die SSRI-Medikation vorher bekannt ([2], S. 44 f.). „Wenn kontrollierte Medikation erlaubt wird, ist es besser möglich, die Piloten zu überwachen. Dadurch wird das Prinzip Selbstauskunft von Piloten gestärkt, da sie ihre Depression angeben können ohne Furcht, dass sie für eine lange Zeit nicht mehr fliegen dürfen. Das wirkt auch der Möglichkeit entgegen, dass Piloten weiterhin mit oder ohne entsprechende Medikamente fliegen, wenn sie an einer Depression leiden.“ Deshalb empfiehlt die BEA, die „Modalitäten zu definieren, unter denen die europäischen Vorschriften es den Piloten erlauben würden als flugmedizinisch tauglich erklärt $\mathrm{zu}$ werden, auch wenn sie Antidepressiva unter ärztlicher Aufsicht einnehmen" ([2], S. 113).

Dementsprechend haben Luftfahrtunternehmen vertrauensfördernde „Maßnahmen zur Erhöhung der Sicherheit" entwickelt. $\mathrm{Zu}$ diesen zählen u. a.:

\section{Kollegiale Unterstützungsprogramme.}

Es sollen kollegiale Unterstützungsprogramme und eine Umgebung gefördert werden, in der Informationen vertraulich behandelt werden, um persönliche und psychische Gesundheitsprobleme zu besprechen.

Unterstützung für Piloten. Piloten sollen unterstützt und geleitet werden. Ziel ist es, Ihnen Hilfe zukommen zu lassen und ggf. die Wiederaufnahme des Flugdiensts zu ermöglichen.

Loss-of-Licence-Versicherung. Die betriebliche Loss-of-Licence-Versicherung ist bei Lufthansa ein integrierter Teil des Tarifvertrags und sieht eine Frühberentung vor, wenn ein Pilot seine Lizenz verliert.

Klare und universell akzeptierte Richtlinien für Fälle, in denen die ärztliche Pflicht zur Meldung flugmedizinischer Bedenken stärker wiegt als die ärztliche Schweigepflicht, also

- bei anzeigepflichtigen Infektionskrankheiten,

- bei klar evidentem Risiko für die öffentliche Sicherheit und dann

- ohne rechtliches Risiko für den Arzt, wenn er z. B. nach französischem StGB ( $\$ 122$, Abs. 7) alle notwendigen und im Vergleich zu der Schwere der Gefahr verhältnismäßigen Schritte zum Schutze der gefährdeten Person unternommen hat (also Bruch der Schweigepflicht nur als letzte Möglichkeit bei Versagen aller anderen Maßnahmen [2], S. 119).

Allerdings sind psychische Erkrankungen hinsichtlich der allgemeinen Gefährdung den genannten Infektionskrankheiten nicht vergleichbar und zu Recht auch nicht anzeigepflichtig. Überdies ist an die im Vergleich zur eindeutigen Diagnose 
einer Infektionskrankheit beschriebenen Schwierigkeiten zu erinnern, die Erheblichkeit einer Gefährdungsabsicht bei einem psychisch Kranken im konkreten Fall evident, d. h. eindeutig festzustellen.

Zwei-Personen-Regelung. Die inzwischen von fast allen Airlines eingeführte "Zwei-Personen-Regelung“, d.h., dass immer zwei autorisierte Personen gleichzeitig im Cockpit sein müssen, scheint die praktikabelste externe Minimierung des Risikos zu sein. Sie sollte durch psychiatrische Maßnahmen wie die gleichsam interne therapeutische Risikominimierung komplettiert werden und müsste im akuten Notfall durch Einweisung bzw. Zwangseinweisung in eine psychiatrische Abteilung ergänzt werden (s. dazu die Stellungnahme „Achtung der Selbstbestimmung und Anwendung von Zwang bei der Behandlung von psychisch erkrankten Menschen" der DGPPN [4]).

Möglicherweise hätte die Katastrophe vermieden werden können, wenn der behandelnde Psychiater die Arbeitsunfähigkeits(AU)-Bescheinigung direkt an den Arbeitgeber zu schicken gehabt hätte. Solche Regelung gibt es aber in Deutschland nicht, weil Patienten die AU-Bescheinigung selbst dem Arbeitgeber übermitteln - und auch ein Interesse daran haben, da sie sonst keine Lohnfortzahlung erhalten. Dass sich ein Angestellter krankschreiben lässt, die AU-Bescheinigung dann aber wie der Kopilot des Germanwings-Flugs zerreißt und weiter zur Arbeit geht, ist offenbar extrem selten, jedoch ein Hinweis darauf, dass gerade bei einer psychischen Erkrankung das Prinzip der Selbstauskunft - als Grundlage der Sicherheitsüberlegungen für Piloten - beeinträchtigt sein kann ([2], S. 109). Deshalb wäre zu prüfen, ob und unter welchen Umständen und in welcher Form die Arbeitsunfähigkeitsbescheinigung in solchen Fällen auch direkt vom Arzt an den Arbeitgeber gegeben werden kann - wie dies in ähnlicher Weise in einigen anderen Ländern geregelt ist.

Richtlinien für alle Gesundheitsdienstleister. Schließlich empfiehlt der BEABericht ([2], S. 116): „Das BMVI und die Bundesärztekammer (BÄK) sollten, ohne auf Maßnahmen auf EU Ebene zu warten, die Richtlinien für alle Gesundheitsdienstleister herausgeben, die:

- sie daran erinnern, dass sie die ärztliche Schweigepflicht brechen und das LBA oder eine andere Behörde informieren können, wenn die Gesundheit eines Verkehrspiloten möglicherweise die öffentliche Sicherheit gefährdet,

- Definitionen für die Begriffe ,bevorstehende Gefahr' und ,Bedrohung der öffentlichen Sicherheit', im Zusammenhang mit der Gesundheit von Piloten enthalten,

- die juristischen Konsequenzen für Gesundheitsdienstleister begrenzen, wenn sie die ärztliche Schweigepflicht, im guten Glauben die Bedrohung der öffentlichen Sicherheit $\mathrm{zu}$ verringern oder $\mathrm{zu}$ eliminieren, verletzten.“

Denn die für einen Piloten existenzgefährdenden Folgen einer Offenbarung seiner psychischen Krankheit könnten Anlass für erhebliche Schadensersatzforderungen sein.

\section{Fazit}

Die Schweigepflicht ist als wesentliche Voraussetzung einer vertrauensvollen Beziehung zwischen Patient und Arzt nicht nur ein hohes Gut, sondern auch wichtigstes (internes) Instrument zur Minimierung von Risiken, die von einem psychisch Kranken ausgehen können. Deshalb kann nach Erfolglosigkeit aller anderen (externen) Maßnahmen zur Abwehr einer erheblichen und unmittelbar drohenden Gefährdung menschlichen Lebens durch einen psychisch kranken Menschen der Bruch der gesetzlich hoch geschützten ärztlichen Schweigepflicht nur das letzte Mittel sein. Die Kriterien einer unvermeidbaren Offenbarung müssen eindeutig definiert und international akzeptabel vereinheitlicht werden. Zudem gilt es, die Organisation der gesundheitlichen Kontrolle der Piloten einschließlich der gegebenenfalls erforderlichen Meldeverfahren noch zu verbessern.

\section{Korrespondenzadresse}

\section{Prof. Dr. H. Helmchen}

Klinik für Psychiatrie und Psychotherapie, Charité - Universitätsmedizin Berlin, Campus Benjamin Franklin

Hindenburgdamm 30, 12200 Berlin,

Deutschland

hanfried.helmchen@charite.de

Interessenkonflikt. H. Helmchen gibt an, dass kein Interessenkonflikt besteht.

\section{Literatur}

1. Appelbaum P (1985) Tarasoff and the clinician: problems in fulfilling the duty to protect. Am J Psychiatry 142:425-429

2. Bureau d'Enquêtes et d'Analyses pour la sécurité de l'aviation civile (BEA) (2016) Abschlussbericht (GermanWings-Unfall). https://www.bea.aero/ uploads/tx_elyextendttnews/BEA2015-0125.deLR_04.pdf.Zugegriffen: 9.Juli 2016

3. Deutsche Gesellschaft für Psychiatrie und Psychotherapie Psychosomatik und Nervenheilkunde (DGPPN) (2015) Stellungnahme zum Absturz von Flug 4U9525. https://www. dgppn.de/fileadmin/user_upload/_medien/ download/pdf/stellungnahmen/2015/2015-0402_Stellungnahme_DGPPN_BVDN_BVDP_Flug_ 4U9525_final.pdf.Zugegriffen:30. März 2016

4. Deutsche Gesellschaft für Psychiatrie und Psychotherapie Psychosomatik und Nervenheilkunde (DGPPN) (Task Force „Ethik in Psychiatrie und Psychotherapie"), Vollmann J, Walter H et al (2014) Achtung der Selbstbestimmung und Anwendung von Zwang bei der Behandlung von psychisch erkrankten Menschen. Eine ethische Stellungnahme der DGPPN. Nervenarzt 85:1419-1431

5. Edwards GS (2010) Doing their duty: an empirical analysis of the unintended effect of Tarasoff $v$ Regents on homicidal activity. https://editorialexpress.com/cgi-bin/conference/ download.cgi?db_name=ALEA2010\&paper_ $\mathrm{id}=87$. Zugegriffen: 3 . Apr. 2016

6. Felthous AR, O'Shaughnessy R, Kuten J et al (2008) The Clinician's Duty to Warn or Protect: In the United States, England, Canada, New Zealand, France and Spain. In: Felthous AR, SaßH (Hrsg) The International Handbook of Psychopathic Disorders and the Law. John Wiley \& Sons, Chichester, NY, S75-94

7. Glasser JM (2012) The Eighteen-Day Running Mate: McGovern, Eagleton, and a Campaign in Crisis. Yale University Press, New Haven

8. Klinka E (2009) It's been a privilege: advising patients of the Tarasoff duty and its legal consequences for the federal psychotherapistpatient privilege. Fordham L Rev 78:863-931

9. Rosenhan DL, Teitelbaum TW, Teitelbaum KW et al (1993) Warning third parties: the ripple effects of Tarasoff. Pac Law J 24:1165-1232

10. Supreme Court of California (SCOCAL) (1976) Tarasoff v. Regents of University of California. (Cal.3d 425) http://scocal.stanford.edu/opinion/ tarasoff-v-regents-university-california-3027817

11. Wise TP (1978) Where the public peril begins: a survey of Psychotherapists to determine the effects of Tarasoff. Stanford Law Rev 31:165-190 
Hier steht eine Anzeige.

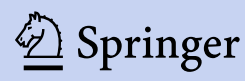

